\title{
P-Stable Obrechkoff Methods with Minimal Phase-Lag for Periodic Initial Value Problems
}

\author{
By U. Ananthakrishnaiah
}

\begin{abstract}
In this paper $P$-stable methods of $O\left(h^{6}\right)$ and $O\left(h^{8}\right)$ with minimal phase-lag (frequency distortion) are derived. Numerical results for both linear and nonlinear problems are presented.
\end{abstract}

1. Introduction. The development of numerical integration formulae for the direct integration of periodic initial value problems

$$
y^{\prime \prime}(t)=f(t, y), \quad y\left(t_{0}\right)=y_{0}, \quad y^{\prime}\left(t_{0}\right)=y_{0}^{\prime}
$$

has created considerable interest in recent years. The numerical integration formulae for (1.1) can be divided into two distinct classes: (a) problems for which the solution period is known in advance; (b) problems for which the period is not known.

Computational methods have been proposed by Gautschi [7], Stiefel and Bettis [12] and Jain et al. [10] which can be used to determine the solution to problems of type (a). In 1976, Lambert and Watson [11] originated the concept of the interval of periodicity and connected it with the symmetry property of the linear multistep method

$$
\sum_{j=0}^{K} \alpha_{j} y_{n+j}=h^{2} \sum_{j=0}^{K} \beta_{j} f_{n+j}
$$

for the development of numerical integration formulae of type (b) for solving (1.1). The characteristic polynomials of (1.2) may be written as

$$
\rho(\xi)=\sum_{j=0}^{K} \alpha_{j} \xi^{j}, \quad \sigma(\xi)=\sum_{j=0}^{K} \beta_{j} \xi^{j} .
$$

The order of the $(\rho, \sigma)$ method (1.2) is defined to be $p$ if for an adequately smooth arbitrary test function $z(x)$,

$$
\sum_{j=0}^{K} \alpha_{j} z(x+j h)-h^{2} \sum_{j=0}^{K} \beta_{j} z^{\prime \prime}(x+j h)=C_{p+2} h^{p+2} z^{p+2}(x)+O\left(h^{p+3}\right)
$$

Received November 16, 1984; revised May 28, 1986 and December 24, 1986. 1980 Mathematics Subject Classification (1985 Revision). Primary 65L05.

Kev words and phrases. $P$-stable, Obrechkoff, phase-lag, periodic initial value problems, second-order differential equations, undamped Duffing's equation. 
where $C_{p+2}$ is the error constant. The $(\rho, \sigma)$ method is assumed to satisfy the following hypotheses (see [11]):

(i) $\alpha_{K}=1,\left|\alpha_{0}\right|+\left|\beta_{0}\right| \neq 0, \sum_{j=0}^{K}\left|\beta_{j}\right| \neq 0$,

(ii) $\rho$ and $\sigma$ have no common factors,

(iii) $\rho(1)=\rho^{\prime}(1)=0, \rho^{\prime \prime}(1)=2 \sigma(1)$;

this is necessary and sufficient for the $(\rho, \sigma)$ method to be consistent, i.e., to have order at least one.

(iv) The method $(\rho, \sigma)$ is zero-stable.

The $(\rho, \sigma)$ method is called symmetric if

$$
\alpha_{j}=\alpha_{K-j}, \quad \beta_{j}=\beta_{K-j} \quad \text { for } j=0,1, \ldots, K .
$$

Applying the method (1.2) to the test equation

$$
y^{\prime \prime}=-\lambda^{2} y, \quad \lambda, y \in \mathbf{R}
$$

and denoting the characteristic equation by

$$
\Omega\left(\xi, H^{2}\right)=\rho(\xi)+H^{2} \sigma(\xi)=0, \quad H=\lambda h,
$$

the following definitions are enunciated by Lambert and Watson [11]:

Definition 1.1. The method $(\rho, \sigma)$ is said to have interval of periodicity $\left(0, H_{0}^{2}\right)$ if for all $H^{2} \in\left(0, H_{0}^{2}\right)$ the roots $\xi_{s}$ of $\Omega\left(\xi, H^{2}\right)$ satisfy

$$
\xi_{1}=e^{i \theta(H)}, \quad \xi_{2}=e^{-i \theta(H)}, \quad\left|\xi_{s}\right| \leqslant 1, s=3,4, \ldots, K,
$$

for some real-valued function $\theta(H)$.

Definition 1.2. The $(\rho, \sigma)$ method (1.2) is said to be P-stable if its interval of periodicity is $(0, \infty)$.

They proved that a necessary and sufficient condition for the $(\rho, \sigma)$ method to have a nonvanishing interval of periodicity is that the method be symmetric and also proved that the order of a $P$-stable $(\rho, \sigma)$ method cannot exceed two.

However, higher-order $P$-stable methods have been discussed by Hairer [9], Cash [3], and Chawla [4] by introducing off-step points or higher-order derivatives of $f(t, y)$ in (1.2).

Gladwell and Thomas [8] have analyzed the conditions which ensure an oscillatory numerical solution. They noted that the symmetric linear multistep methods proposed by Lambert and Watson [11] have no algorithmic damping, but do not perform well for problems with high frequency natural nodes. They also noted that methods with minimal phase-lag (frequency distortion) error perform better than the method with no algorithmic damping. They also considered a slightly more general test equation

$$
y^{\prime \prime}+\lambda^{2} y=\nu e^{i \omega t}
$$

to derive conditions which ensure that the numerical forced oscillation is in phase with the true forced oscillation. Later, Thomas [13] determined phase-lag errors for various $P$-stable methods proposed by Lambert and Watson [11], Cash [3] and Chawla [4].

Following Gladwell and Thomas [8], Chawla and Rao [5] and Ananthakrishnaiah [1] developed two-step methods with minimal phase-lag errors $\lambda^{6} h^{6} / 12096$ and $\lambda^{6} h^{6} / 42000$, respectively. The minimal phase-lag method of Chawla and Rao [5] is of 
$O\left(h^{4}\right)$ with interval of periodicity $(0,2.71)$ whereas the method of Ananthakrishnaiah [1] is of $O\left(h^{2}\right)$ and is $P$-stable. The derivations depend upon the definition for phase-lag error given by Brusa and Nigro [2].

Definition 1.3. The phase-lag of a numerical method is the leading coefficient of

$$
\left|\frac{\theta(H)-H}{H}\right|
$$

where $\tan \theta(H)=[A(H)-B(H)]^{1 / 2} / B(H), A(H), B(H)$ being given by the characteristic polynomial

$$
\Omega(\xi, H)=A(H) \xi^{2}-2 B(H) \xi+A(H) .
$$

In this article we consider symmetric two-step Obrechkoff methods involving higher-order derivatives in the form

$$
\delta^{2} y_{n}=\sum_{i=1}^{m} h^{2 i}\left[\beta_{i 0} y_{n+1}^{(2 i)}+2 \beta_{i 1} y_{n}^{(2 i)}+\beta_{i 0} y_{n-1}^{(2 i)}\right] .
$$

It is noted that one can easily derive an $O\left(h^{2}\right)$ method (Dahlquist method; see [6]), $O\left(h^{4}\right)$ and $O\left(h^{6}\right) P$-stable methods with minimal phase-lag errors by using the definition (1.8), but it will not yield us an $O\left(h^{8}\right) P$-stable method with minimal phase-lag error. So the motivation of this article is to derive and implement the definition for phase-lag error and the conditions for obtaining $P$-stable methods up to $O\left(h^{8}\right)$ with minimal phase-lag errors. Since one can easily verify that the definition and conditions introduced in this article produce the same $P$-stable methods of $O\left(h^{2}\right)$ and $O\left(h^{4}\right)$ as the definition (1.8) (for $m=1,2$ in (1.10)), we concentrate on presenting $P$-stable methods of $O\left(h^{6}\right)$ and $O\left(h^{8}\right)$ with minimal phase-lag errors using the symmetric multi-derivative two-step methods given by (1.10). The methods developed are applied to a test problem and to the nonlinear undamped Duffing's equation which has forced oscillations in its true solution, and hence it is desirable that the numerical solution is also in phase with the oscillatory true solution (see [8]).

2. Derivation of the P-Stable Methods of $O\left(h^{6}\right)$ and $O\left(h^{8}\right)$ with Minimal Phase-Lag. Applying the method (1.10) to the test equation (1.6), we obtain the characteristic polynomial

$$
\Omega(\xi, H)=A(H) \xi^{2}-2 B(H) \xi+A(H)
$$

where

$$
A(H)=1+\sum_{i=1}^{m}(-1)^{i-1} \beta_{i 0} H^{2 i}, \quad B(H)=1-\sum_{i=1}^{m}(-1)^{i-1} \beta_{i 1} H^{2 i},
$$

with $H^{2}=\lambda^{2} h^{2}$.

The roots of (2.1) are complex and of modulus one if

$$
\left|\frac{B(H)}{A(H)}\right|<1 \text {. }
$$

Let the roots of (2.1) be

$$
\xi_{1,2}=e^{ \pm i \theta(H)}
$$


when (2.2) is satisfied. The exact solution of the test equation (1.6) with the initial conditions $y\left(t_{0}\right)=y_{0}$ and $y^{\prime}\left(t_{0}\right)=y_{0}^{\prime}$ is given by

$$
y(t)=y_{0} \cos \lambda t+\frac{y_{0}^{\prime}}{\lambda} \sin \lambda t
$$

Evaluating (2.3) at $t_{n+1}, t_{n}$ and $t_{n-1}$ and eliminating $y_{0}$ and $y_{0}^{\prime}$, we obtain

$$
y\left(t_{n+1}\right)-2 \cos \lambda h y\left(t_{n}\right)+y\left(t_{n-1}\right)=0,
$$

whose characteristic equation is

$$
\xi^{2}-2 \cos H \xi+1=0, \quad H=\lambda h .
$$

The characteristic equation of (2.1) may be written as

$$
\xi^{2}-2 \cos \theta(H) \xi+1=0,
$$

where $\cos \theta(H)=B(H) / A(H)$.

Definition 2.1. We define the phase-lag error of the method (1.10) as the leading coefficient in the expansion of

$$
\left|\frac{A(H) \cos (H)-B(H)}{H^{2}}\right|
$$

and denote it by $P(H)$. (The motivation of this definition may be easily noted to be the difference in the frequency distortion of the characteristic equations (2.6) and (2.5).)

Taking $m=4$ and using (2.7) we obtain

$$
\begin{aligned}
P(H)= & \left(\beta_{10}+\beta_{11}-\frac{1}{2}\right)-\left(\beta_{20}+\beta_{21}+\frac{\beta_{10}}{2}-\frac{1}{24}\right) H^{2} \\
& +\left(\beta_{30}+\beta_{31}+\frac{\beta_{20}}{2}+\frac{\beta_{10}}{24}-\frac{1}{720}\right) H^{4} \\
& -\left(\beta_{40}+\beta_{41}+\frac{\beta_{30}}{2}+\frac{\beta_{20}}{24}+\frac{\beta_{10}}{720}-\frac{1}{8 !}\right) H^{6} \\
& +\left(\frac{\beta_{40}}{2}+\frac{\beta_{41}}{2}+\frac{\beta_{30}}{24}+\frac{\beta_{20}}{720}+\frac{\beta_{10}}{8 !}-\frac{1}{10 !}\right) H^{8}+O\left(H^{10}\right) .
\end{aligned}
$$

The $P$-stability condition (2.2) is satisfied if $A+B>0$ and $A-B>0$, where

$$
\begin{aligned}
A+B= & 2+\left(\beta_{10}-\beta_{11}\right) H^{2}-\left(\beta_{20}-\beta_{21}\right) H^{4}+\left(\beta_{30}-\beta_{31}\right) H^{6} \\
& -\left(\beta_{40}-\beta_{41}\right) H^{8}, \\
A-B= & \left(\beta_{10}+\beta_{11}\right) H^{2}\left[1-\frac{\beta_{20}+\beta_{21}}{\beta_{10}+\beta_{11}} H^{2}+\frac{\beta_{30}+\beta_{31}}{\beta_{10}+\beta_{11}} H^{4}\right. \\
& \left.-\frac{\beta_{40}+\beta_{41}}{\beta_{10}+\beta_{11}} H^{6}\right] .
\end{aligned}
$$

In order that the phase-lag error be of $O\left(H^{8}\right)$, we obtain the following order conditions to be satisfied:

$$
\begin{aligned}
& \beta_{10}+\beta_{11}=\frac{1}{2}, \\
& \beta_{20}+\beta_{21}+\frac{\beta_{10}}{2}=\frac{1}{24}, \\
& \beta_{30}+\beta_{31}+\frac{\beta_{20}}{2}+\frac{\beta_{10}}{24}=\frac{1}{720},
\end{aligned}
$$




$$
\beta_{40}+\beta_{41}+\frac{\beta_{30}}{2}+\frac{\beta_{20}}{24}+\frac{\beta_{10}}{720}=\frac{1}{8 !} .
$$

One can easily verify that the solution satisfying (2.10) and the $P$-stability conditions (2.9.1) and (2.9.2) is

$$
\begin{aligned}
& \beta_{10}=\frac{1}{28}, \quad \beta_{11}=\frac{13}{28}, \quad \beta_{20}=\frac{-9}{11760}, \quad \beta_{21}=\frac{289}{11760}, \\
& \beta_{30}=\frac{1}{70560}, \quad \beta_{31}=\frac{19}{70560}, \quad \beta_{40}=\frac{11}{2822400}=-\beta_{41} .
\end{aligned}
$$

Hence the method

$$
\begin{aligned}
\delta^{2} y_{n}= & \frac{h^{2}}{28}\left(y_{n+1}^{\prime \prime}+26 y_{n}^{\prime \prime}+y_{n-1}^{\prime \prime}\right)-\frac{h^{4}}{11760}\left(9 y_{n+1}^{\mathrm{iv}}-578 y_{n}^{\mathrm{iv}}+9_{n-1}^{\mathrm{iv}}\right) \\
& +\frac{h^{6}}{70560}\left(y_{n+1}^{\mathrm{vi}}+38 y_{n}^{\mathrm{vi}}+y_{n-1}^{\mathrm{vi}}\right) \\
& -\frac{h^{8}}{2822400}\left(y_{n+1}^{\mathrm{viii}}-2 y_{n}^{\mathrm{viii}}+y_{n-1}^{\mathrm{viii}}\right)
\end{aligned}
$$

with the truncation error

$$
\mathrm{TE}=\frac{-2 h^{10} y^{(10)}}{7 \cdot(10 !)}+O\left(h^{12}\right)
$$

is a $P$-stable method of $O\left(h^{8}\right)$ with the minimal phase-lag error

$$
P(H)=\frac{H^{8}}{7 \cdot(10 !)} .
$$

Taking $m=3$ and using (2.7), we obtain the method

$$
\begin{aligned}
\delta^{2} y_{n}= & \frac{h^{2}}{20}\left(y_{n+1}^{\prime \prime}+18 y_{n}^{\prime \prime}+y_{n-1}^{\prime \prime}\right)-\frac{h^{4}}{600}\left(y_{n+1}^{\mathrm{iv}}-22 y_{n}^{\mathrm{iv}}+y_{n-1}^{\mathrm{iv}}\right) \\
& +\frac{h^{6}}{14400}\left(y_{n+1}^{\mathrm{vi}}+2 y_{n}^{\mathrm{vi}}+y_{n-1}^{\mathrm{vi}}\right)
\end{aligned}
$$

with the truncation error

$$
\mathrm{TE}=\frac{h^{8} y_{n}^{\mathrm{vii}}}{50400}+O\left(h^{10}\right),
$$

which is a $P$-stable method of order six with minimal phase-lag $P(H)=H^{6} / 2 .(7 !)$.

3. Numerical Results. We consider the test problem

$$
y^{\prime \prime}=-\lambda^{2} y, \quad y(0)=1, \quad y^{\prime}(0)=0
$$

with $\lambda^{2}=100$. Using the step length $h=\pi / 12$, the absolute errors in the solution $y(t)$ are tabulated in Table 1 for $t=2 \pi(2 \pi) 10 \pi$. We consider the nonlinear undamped Duffing's equation

$$
y^{\prime \prime}+y+y^{3}=B \cos \Omega t
$$


with $B=0.002, \Omega=1.01$. The exact solution (see [14]) is given by

$$
y(t)=A_{1} \cos \Omega t+A_{3} \cos 3 \Omega t+A_{5} \cos 5 \Omega t+A_{7} \cos 7 \Omega t
$$

where

$$
\begin{aligned}
& A_{1}=0.200179477536, \\
& A_{3}=0.246946143(-03), \\
& A_{5}=0.304016(-06), \\
& A_{7}=0.374(-09) .
\end{aligned}
$$

To find the values of $y^{\prime}$ which occur in calculating $y^{\text {iv }}$ and $y^{\text {vi }}$, we use the $O\left(h^{4}\right)$ differentiation formula given by

$$
y_{n+1}^{\prime}=\frac{1}{2 h}\left(3 y_{n+1}-4 y_{n}+y_{n-1}\right)+\frac{h}{12}\left(3 y_{n+1}^{\prime \prime}-2 y_{n}^{\prime \prime}-y_{n-1}^{\prime \prime}\right)+O\left(h^{4}\right) .
$$

The implicit methods (2.11) and (2.10) are implemented using Picard iteration with error tolerance $\varepsilon=1.0 \mathrm{E}-10$. Taking the steplength $h=\pi / 5$, the absolute errors for for $t=2 \pi(2 \pi) 10 \pi$ are presented in Table 2 .

\section{TABLE 1}

Absolute errors in $y(t)$ for the problem (3.1) with $h=\pi / 12$

\begin{tabular}{rccc}
\hline$t$ & Method (2.11) & Method (2.12) & Hairer's Method \\
\hline$\pi$ & $2.06(-06)$ & $2.40(-03)$ & $6.49(-01)$ \\
$2 \pi$ & $9.08(-06)$ & $1.05(-02)$ & $1.82(00)$ \\
$4 \pi$ & $3.80(-05)$ & $4.38(-02)$ & $5.66(-01)$ \\
$6 \pi$ & $8.67(-05)$ & $9.91(-02)$ & $9.35(-01)$ \\
$8 \pi$ & $1.55(-04)$ & $1.75(-01)$ & $1.55(00)$ \\
$10 \pi$ & $2.43(-04)$ & $2.70(-01)$ & $1.10(-01)$ \\
\hline
\end{tabular}

TABLE 2

Absolute errors in $y(t)$ for the problem (3.2) with $h=\pi / 5$

\begin{tabular}{rcc}
\hline$t$ & Method (2.12) & Hairer's method \\
\hline$\pi$ & $4.53(-05)$ & $5.03(-02)$ \\
$2 \pi$ & $1.88(-04)$ & $4.79(-02)$ \\
$4 \pi$ & $7.46(-04)$ & $4.25(-02)$ \\
$6 \pi$ & $1.63(-03)$ & $3.64(-02)$ \\
$8 \pi$ & $2.78(-03)$ & $2.97(-02)$ \\
$10 \pi$ & $4.11(-03)$ & $2.26(-02)$ \\
\hline
\end{tabular}

4. Acknowledgment. This research work is supported by the National Science and Engineering Research Council operating grants of Professors R. Manohar and J. W. Stephenson. The author is grateful to the referee for his helpful suggestions, which improved the presentation of the article.

Department of Mathematics 
1. U. Ananthakrishnaiah, "A class of two-step $P$-stable methods for the accurate integration of second order periodic initial value problems," J. Comput. Appl. Math., v. 14, 1986, pp. 455-459.

2. L. BRUSA \& L. Nigro, "A one-step method for direct integration of structural dynamic equations," Internat. J. Numer. Methods Engrg., v. 15, 1980, pp. 685-699.

3. J. R. CASH, "High order $P$-stable formulae for the numerical integration of periodic initial value problems," Numer. Math., v. 37, 1981, pp. 355-370.

4. M. M. Chawla, "Two-step fourth order $P$-stable methods for second order differential equations," $B I T$, v. 21, 1981, pp. 190-193.

5. M. M. ChaWla \& P. S. RaO, "A Numerov-type method with minimal phase-lag for the integration of second order periodic initial value problems," J. Comput. Appl. Math., v. 11, 1984, pp. 277-281.

6. G. DAhlQuist, "On accuracy and unconditional stability of linear multistep methods for second order differential equations," BIT, v. 18, 1978, pp. 133-136.

7. W. GautsChI, "Numerical integration of ordinary differential equations based on trigonometric polynomials," Numer. Math., v. 3, 1961, pp. 381-397.

8. I. Gladwell \& R. M. Thomas, "Damping and phase analysis for some methods for solving second order ordinary differential equations," Internat. J. Numer. Methods Engrg., v. 19, 1983, pp. 493-503.

9. E. HaIreR, "Unconditionally stable methods for second order differential equations," Numer. Math., v. 32, 1979, pp. 373-379.

10. M. K. Jain, R. K. Jain \& U. Ananthakrishnaiah, " $P$-stable methods for periodic initial value problems of second order differential equations," BIT, v. 19, 1979, pp. 347-355.

11. J. D. LamberT \& I. A. Watson, "Symmetric multistep methods for periodic initial value problems," J. Inst. Math. Appl., v. 18, 1976, pp. 189-202.

12. E. Stiefel \& D. G. BetTis, "Stabilization of Cowell's methods," Numer. Math., v. 13, 1969, pp. $154-175$

13. R. M. Thomas, "Phase properties of high order almost $P$-stable formulae," BIT, v. 24, 1984, pp. 225-238.

14. R. Van Dooren, "Stabilization of Cowell's classical finite difference method for numerical integration," J. Comput. Phys., v. 16, 1974, pp. 186-192. 